\title{
Several faces of multiple myeloma
}

\author{
Maria do Mar Menezes ${ }^{1}$, Elsa Soares ${ }^{2}$, Mário Góis ${ }^{1,3,4}$, Helena Viana ${ }^{1,3,4}$, Fernando Nolasco ${ }^{1,4}$ \\ ${ }^{1}$ Nephrology Department, Hospital de Curry Cabral, Centro Hospitalar e Universitário de Lisboa Central, Lisbon Portugal \\ ${ }^{2}$ Nephrology Department, Centro Hospitalar de Setúbal, Setúbal, Portugal \\ ${ }^{3}$ Laboratory of Renal Morphology, Nephrology Department, Hospital Curry Cabral - Centro Hospitalar e Universitário de Lisboa Central, Lisbon, Portugal \\ ${ }^{4}$ Nova Medical School, Faculdade de Ciências Médicas, Universidade Nova de Lisboa, Lisbon, Portugal
}

\section{CLINICAL PRESENTATION}

We present a case of a 71-year-old woman with past medical history of hypertension and multiple myeloma (MM) IgG kappa for three years. She underwent four chemotherapy lines (vincristine, doxorubicin and dexamethasone; thalidomide plus dexamethasone; bortezomib, cyclophosphamide and dexamethasone and lenalidomide plus dexamethasone). Five months ago, she started a $5^{\text {th }}$ therapeutic line with carfilzomib and dexamethasone. But several infections delayed the correct periodicity.

She was admitted to the Oncology Department with bilateral pneumoniae caused by Influenza A and syncytial respiratory virus. She was treated empirically with oseltamivir and piperacillin tazobactam for 7 days. Chemotherapy was deferred. Initial blood analysis revealed pancytopenia with $\mathrm{Hbg} 8 \mathrm{~g} / \mathrm{dL}$, platelets 31,000 and leucocytes 2,800, c-reactive protein $310 \mathrm{mg} / \mathrm{L}, \mathrm{sCr} 0.8 \mathrm{mg} / \mathrm{dL}$ and serum free light chains (sFLC) kappa $500 \mathrm{mg} / \mathrm{L}$.

At the $7^{\text {th }}$ day she presented with severe hypoxemia and acute renal failure - serum creatinine $4 \mathrm{mg} / \mathrm{dL}$ - and was transferred to the Intensive Care Unit. Given her anuria and metabolic acidosis, she started intermittent hemodialysis. Renal ultrasound was normal. As a complication, the patient had a bacteremia caused by Escherichia coli and was treated with a 7-day course of meropenem. Three weeks later, clinically improved but hemodialysis dependent, she was admitted to the nephrology department to investigate the etiology of her acute renal failure. The workup revealed $\mathrm{Hb} 8 \mathrm{~g} / \mathrm{dL}$, platelets 48,000 , leucocytes $3,000, \mathrm{sCr} 5.6 \mathrm{mg} / \mathrm{dL}$, albumin $2.7 \mathrm{~g} / \mathrm{dL}, \mathrm{Ca}+7.8 \mathrm{mg} / \mathrm{dL}, \mathrm{LDH} 450 \mathrm{U} / \mathrm{L}$, no schistocytes, normal haptoglobin and ADAMTS13 activity, ANA, ANCA, anti-GBM, complement and serologic testing including HIV, hepatitis $C$ and $B$ virus all were negative. The patient recovered diuresis and stopped dialysis temporarily but 5 days later an increase in $\mathrm{sCr}$ to $9 \mathrm{mg} / \mathrm{dL}$, sU $90 \mathrm{mg} / \mathrm{dL}$ with uremic syndrome and oliguria forced us to restart dialysis. At this point, blood tests revealed a drastic increase in sFLC to $2160 \mathrm{mg} / \mathrm{L}$, ratio kappa/lambda 156, IgG 46g/L, immunoparesis IgM and IgA, erythrocyte sedimentation rate $114 \mathrm{~mm} / \mathrm{h}, \mathrm{LDH} 438 \mathrm{U} / \mathrm{L}$ and a suppressed haptoglobin $<0.08 \mathrm{~g} / \mathrm{L}$. A renal biopsy was therefore performed.

\section{QUESTIONS}

1. According to the clinical and laboratory findings, what is the most likely diagnosis?

2. Taking into consideration the renal biopsy, what is the final diagnosis?

3. What treatment options do we have in this case?

4. What is this patient's renal and vital prognosis?

\section{ANSWERS}

1. According to the clinical and laboratory findings, what is the most likely diagnosis?

In view of the clinical evolution of the patient, we assume as an initial hypothesis that it was an acute kidney injury in the context of severe sepsis. Thus, we expect to find only aspects of acute tubular necrosis (ATN) in renal biopsy. The beginning of diuresis supported our hypothesis.

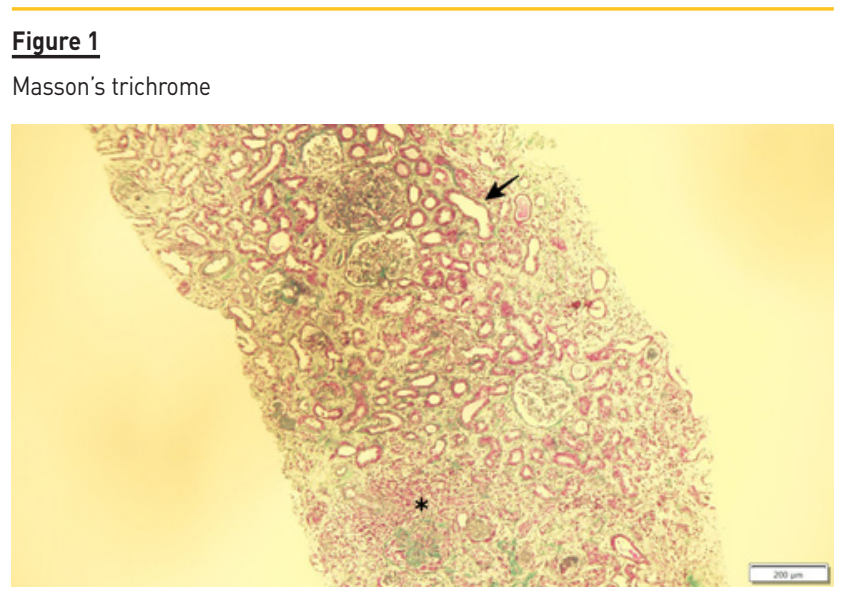

\section{Figure 2}

Periodic Acid-Schiff

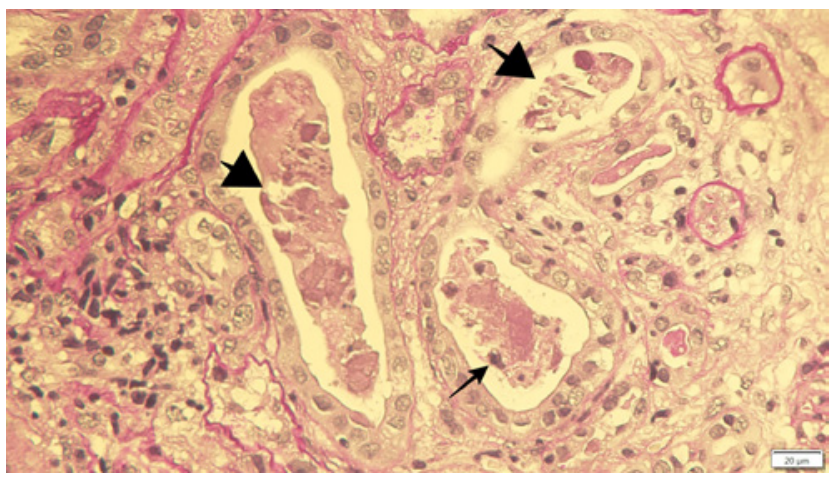




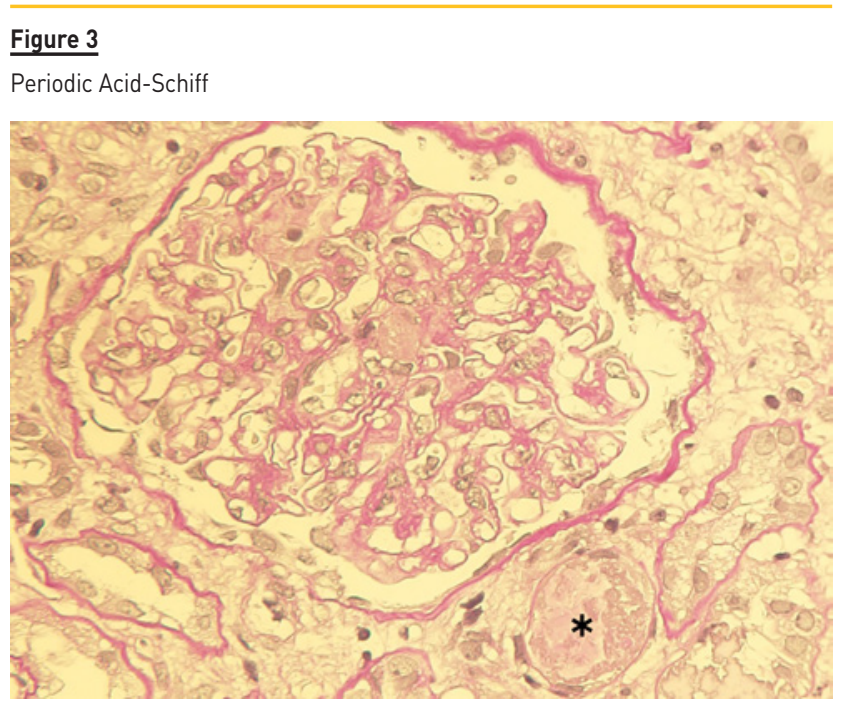

\section{Figure 4}

Periodic Acid-Schiff

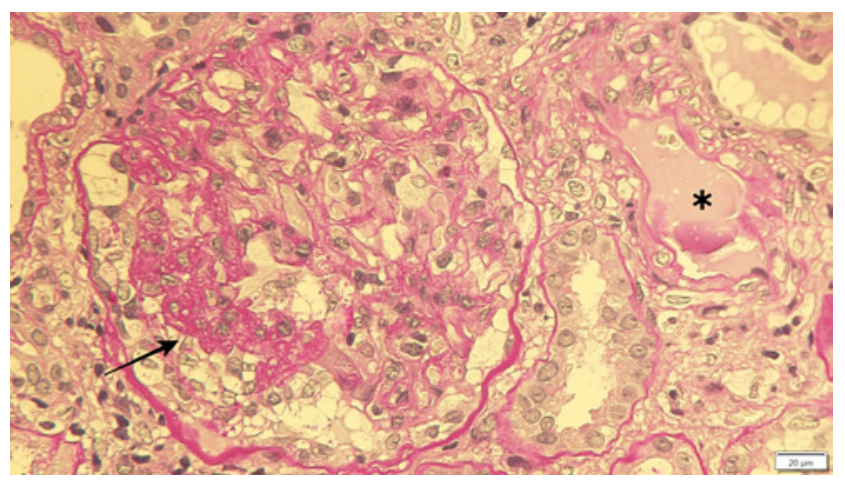

Another hypothesis would be drug toxicity, especially antibiotics. In this case, we would expect to have aspects of interstitial nephritis in the biopsy.

Nevertheless, the patient took long than would be expected to recover from ATN and new data emerged. Despite pancytopenia may have been primarily justified by infection and immunosuppression state; later, the anemia and thrombocytopenia associated with a low haptoglobin and elevated LDH raised the hypothesis of thrombotic microangiopathy (TMA).

TMA is a rare complication in patients with MM under treatment with proteasome inhibitors (PI), particularly carfilzomib and bortezomib ${ }^{1}$. After excluding other causes of TMA, we assumed it was secondary to carfilzomib. There are some case reports of late TMA in the literature ${ }^{2,3}$.

On the other hand, progression of $\mathrm{MM}$ is evident as shown by an increase of sFLC from 500 to $2140 \mathrm{mg} / \mathrm{L}$, and renal involvement could be a supposition. Myeloma cast nephropathy is a major cause of renal failure

\section{Figure 5}

Immunofluorescence (kappa and lambda light chains)

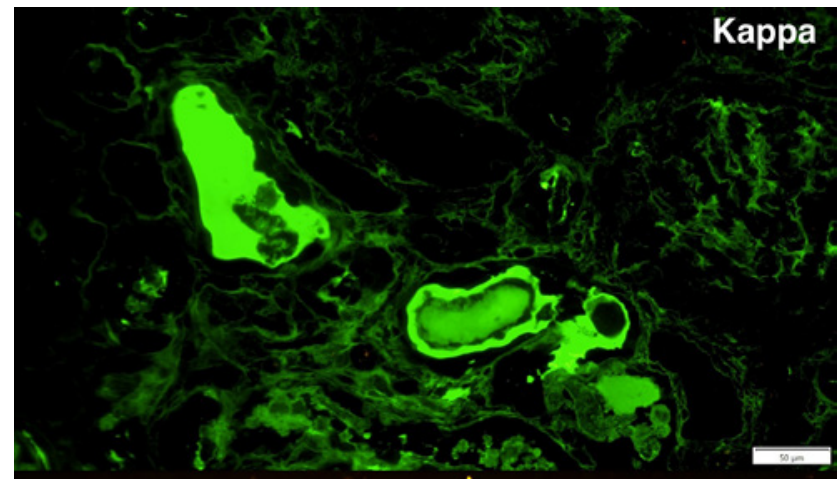

Lambda

and values $>1500 \mathrm{mg} / \mathrm{L}$ are consistent with that hypothesis, but the patient's acute renal failure began before this rise. On the other hand, the tubular obstruction causes a rapid onset of kidney injury, which was the case ${ }^{4}$.

\section{Taking into consideration the renal biopsy, what is the final} diagnosis?

Figure 1 shows alterations compatible with ATN (arrow) and areas with interstitial inflammation $\left({ }^{*}\right)$. In figure 2 , we can observe tubular casts, which exhibit irregular shapes and fracture planes (thick arrows). These casts are PAS negative and are located within the distal tubules. Casts also contain cell debris from tubular damage and are surrounded by multinucleated cell reaction (thin arrow). Figure 3 shows a thrombus within an arteriole $\left({ }^{*}\right)$. Figure 4 represents a thrombus in a small-sized artery $\left({ }^{*}\right)$ and a glomerulus with ischemia of the capillary tuft (arrow). We preformed immunofluorescence on pronase-digested paraffin sections, which showed that casts stained for kappa and were negative for lambda.

Finally, two histologic diagnoses were made: kappa light chain nephropathy and thrombotic microangiopathy (TMA).

\section{What treatment options do we have in this case?}

Taking into account the different disease patterns in renal biopsy, the following changes were made. 
The treatment of acute tubular necrosis is directed to the underlying cause, which in this case were a severe pneumoniae and bacteremia (E. coli). The patient had already been through two antibiotic cycles with clinical improvement.

Cast nephropathy requires immediate treatment. The initial treatment includes vigorous hydration (saline infusion $0.9 \%$ ) to ensure a urine output of $150 \mathrm{~mL} /$ hour and avoid cast formation ${ }^{1}$ Nevertheless, our patient was oliguric and the risk of fluid overload precluded this strategy. All nephrotoxic were avoided (non-steroid anti-inflammatories, angiotensin converting enzyme inhibitors, contrast agents, diuretics). The treatment indicated was to initiate myeloma-directed chemotherapy and dexamethasone was immediately re-started $(10 \mathrm{mg} /$ day).

The extracorporeal methods for light chain removal with high-cutoff hemodialysis membranes in combination with antimyeloma therapy is controversial, as shown by mixed results from MYRE and EULYTE trials ${ }^{1}$. Therefore they were not performed.

TMA has several etiologies, namely primary (which can be further divided into hereditary or acquired), secondary or infection related ${ }^{5}$. In this case, either MM itself or drug-induced TMA were possible explanations. We can identify a causal agent, which has been described in the literature - Carfilzomib. In most reports, TMA occurs after the introduction of this drug, but there are also later cases (up to 2 years). We assumed this was the circumstance.

There are several postulated mechanisms of proteasome inhibitorsinduced TMA. The first one is related to immune-mediated toxicity, assuming the increase of high levels of proinflammatory cytokines (including IL-6 and TNF-a) by PI (proteasome inhibitors) which produce a microvascular environment permitting the development of drugdependent antibodies. The second hypothesis is a dose-mediated toxicity mechanism that involves VEGF inhibition leading to microvascular injury, mostly to glomerular capillaries ${ }^{6}$.

Therefore, a change in this treatment was required and carfilzomib was not restarted.

There are several case reports in the literature with different approaches that include, in addition to the stopping of PI: Plasmapheresis, steroids and eculizumab, with diverse outcomes ${ }^{6}$.

In our case, daratumumab, a CD38+ antibody against plasma cells, was the next step proposed by Hematology.

\section{What is this patient's renal and vital prognosis?}

Multiple myeloma is a plasma cell disorder characterized by abnormal proliferation of plasma cells resulting in overproduction of paraprotein. It is rare and historically believed to be an incurable cancer.

Renal involvement is one of the most common complications of $\mathrm{MM}$ that occurs in $20-50 \%$ of patients, and $10 \%$ to $15 \%$ will require dialysis $^{7,8}$. It can present in a variety of forms (Ig deposition related or not) and it is associated with an increased mortality 7,8 . There are reports that refer a median overall survival of 8.6 months in patients with acute kidney injury whereas patients who did not develop AKI had a median OS of 34.5 months ${ }^{7}$.

In spite of that, improvements in therapy in the last decade have prolonged patient survival.

Patients requiring dialysis have reported rates of renal recovery as low as 5 to $15 \%$ but with new targeted sFLC reduction strategies, these numbers can increase to $75 \%{ }^{9}$.

According to the Revised International Staging System, the survival of these patients depends on the stage of the disease. Our patient was in stage III and would have a mean survival time of 40 months. However, we have to take into consideration that the patient is already undergoing her $5^{\text {th }}$ line of treatment and so obviously this has to be adjusted.

In conclusion, this patient had a poor renal and vital prognosis. Despite normalization of haptoglobin two weeks later, there was no recovery of renal function ( $\mathrm{sCR} 6 \mathrm{mg} / \mathrm{dL}$ ). The patient was discharged dialysis-dependent, and died three weeks later due to another infectious complication.

Disclosure of potential conflicts of interest: none declared

\section{References}

1. Treatment and prognosis of kidney disease in multiple myeloma and other monoclonal gammopathies. Available at https://www.uptodate.com/contents/treatment-and-prognosisof-kidney-disease-in-multiple-myeloma-and-other-monoclonal-gammopathies . Accessed June 6, 2020.

2. Haddadin M, Al-Sadawi M, Madanat S et al. Late presentation of carfilzomib associated thrombotic microangiopathy. Am J Med Case Rep. 2019; 7(10):240-243.

3. Yui J, Keer J, Weiss B et al. Proteasome inhibitor associated thrombotic microangiopathy. Am J Hematol. 2106; 91:E348-E352.

4. Hogan J, Alexander M, Leung N. Dysproteinemia and the kidney: Core curriculum 2019. Am J Kidney Dis. 2019; 74(6):822-836

5. Brocklebank V, Wood K, Kavanagh D. Thrombotic microangiopathy and the kidney. Clin J Am Soc Nephrol. 2008; 13:300-317.

6. Monteith B E, Venner C, Reece D et al. Drug-induced thrombotic microangiopathy with concurrent proteasome inhibitor use in the treatment of multiple myeloma: A case series and review of the literature. Clinical Lymphoma, Myeloma and Leukemia. 2020; Epub ahead print. doi: https://doi. org $/ 10.1016 /$ j.clml.2020.04.014..

7. Leung N, Nasr S. Myeloma-related kidney disease. Adv Chronic Kidney Dis. 2014;21(1):36-47.

8. Dimopoulos $\mathrm{M}$ et al. International myeloma working group recommendations for the diagnosis and management of myeloma-related renal impairment. J Clin Oncol. 2016; 34(13):1544-1557.

9. Johnson RJ, Feehally J, Floege J, Tonelli M. Comprehensive Clinical Nephrology, 6th Edition. Elsevier $08 / 2018$

\section{Correspondence to:}

Mário Góis, MD

Laboratory of Renal Morphology, Hospital Curry Cabral Centro Hospitalar e Universitário de Lisboa Central, Lisboa, Portugal

E-mail: mario.gois@chlc.min-saude.pt 